\title{
PURE STATE EXTENSIONS OF THE TRACE ON THE CHOI ALGEBRA
}

\author{
ALDO LAZAR, SZE-KAI TSUI AND STEPHEN WRIGHT
}

(Communicated by John B. Conway)

\begin{abstract}
Pure state extensions of a nonhyperfinite $\mathrm{II}_{1}$-factor state on the Choi algebra to the Cuntz algebra $\mathrm{O}_{2}$ is constructed.
\end{abstract}

1. Introduction. Let $\mathbf{A}$ be a $C^{*}$-algebra. A state $\varphi$ of $\mathbf{A}$ is a factor state if the closure of the image of $\mathbf{A}$ under the GNS representation induced by $\varphi$ in the weak operator topology is a factor. We call this the image factor induced by $\varphi$. We will say that $\varphi$ is a factor state of type $\mathrm{I}, \mathrm{II}_{1}, \mathrm{II}_{\infty}$, or III if the image factor induced by $\varphi$ is, respectively, type $\mathrm{I}, \mathrm{II}_{1}, \mathrm{II}_{\infty}$, or III. If $\mathbf{B}$ is a $C^{*}$-subalgebra of $\mathbf{A}$, an old question in the theory of operator algebras asks if every factor state of $\mathbf{B}$ extends to a factor state of $\mathbf{A}$. This was answered in the affirmative recently for a separable $C^{*}$-subalgebra $\mathbf{B}$ in independent work of Longo [6] and Popa [7]. They each completed a procedure first outlined by Sakai [8] to obtain the desired extensions.

Given the validity of this extension procedure, a natural problem to consider next is the relation, if any, between the type of the image factor induced by the state $\varphi$ of $\mathbf{B}$ and the type of the image factor induced by an extension of $\varphi$ to a factor state $\mathbf{A}$. In a talk given at the conference on Operator Algebras and $K$ theory at the Mathematical Sciences Research Institute at Berkeley in June 1985, R. J. Archbold asked two specific questions related to this problem:

1. For a non-type I factor state of $\mathbf{B}$ can we get a factor state extension to $\mathbf{A}$ of type I, II, or III?

2. If $\varphi$ is a factor state of $\mathbf{B}$, and if $S(\varphi)$ denotes the set of all extensions of $\varphi$ to a state of $\mathbf{A}$, does the set of extreme points of $S(\varphi)$ contain a factor state of $\mathbf{A}$ ?

The Sakai extension technique mentioned before sheds very little light on Questions 1 and 2. In order to make progress on these questions, the development of new factor state extension techniques is therefore necessary. Woronowicz [10] has found a method which extends each factor state of a $C^{*}$-algebra $\mathbf{B}$ to a pure state of $\mathbf{B} \otimes \mathbf{B}^{\text {op }}$ where $\mathbf{B}^{\text {op }}$ is the $C^{*}$-algebra obtained from $\mathbf{B}$ by reversing its multiplication, and $\otimes$ denotes the projective $C^{*}$-tensor product. This is the best possible example of an affirmative answer to question 2 and to the type I conclusion of question 1. In addition to this, Tsui [9] has shown that if $\mathbf{B}$ is the commutant in

Received by the editors September 30, 1985 and, in revised form, December 18, 1986. Part of this paper was presented in a Great Plain Operator Theory Seminar in Cincinnati, May 1986.

1980 Mathematics Subject Classification (1985 Revision). Primary 46L30, 46L35.

Key words and phrases. Factor state extensions, type $\mathrm{II}_{1}$ factors, pure state extension, the reduced $C^{*}$-algebra of a discrete group.

The second author is partially supported by a fellowship from Oakland Research Excellence and Economic Development Fund. 
a $C^{*}$-algebra $\mathbf{A}$ of a finite-dimensional $C^{*}$-subalgebra of $\mathbf{A}$, then Question 2 has an affirmative answer; in fact, every extreme point of $S(\varphi)$ is a factor state in this case.

In this paper, we add to this small list of examples one other by considering the unique, normalized trace on the Choi algebra, a factor state of type $\mathrm{II}_{1}$, and extending it to a pure state of the Cuntz algebra $\mathrm{O}_{2}$. While this result is admittedly very special, the technique involved makes use of the combinatorial features of the group structure of the Choi algebra-Cuntz algebra embedding, and describes how factor state extensions of the trace can be obtained by varying the way in which this group structure is realized spatially. The hope therefore is that a general group-theoretic approach to factor state extensions can be developed to eventually generate examples which illustrate the other possibilities that occur in Question 1. In that spirit, we close this introduction by posing the following problem: Find type $\mathrm{II}_{\infty}$ and type $\mathrm{III}_{\lambda}, 0 \leq \lambda \leq 1, \lambda \neq 1 / 2$, factor state extensions to $O_{2}$ of the trace on the Choi algebra (type $\mathrm{II}_{1}$ factor states of $\mathrm{O}_{2}$ are, of course, not possible since $\mathrm{O}_{2}$ is simple and stable). It was pointed out by C. Lance that the $\mathrm{III}_{1 / 2}$-factor state extension to $\mathrm{O}_{2}$ of the trace on the CAR $C^{*}$-subalgebra of D. Evans [5] is also an extension of the trace on the Choi algebra. (See Example 4.2 in [1].)

ACKNowledgment. We wish to thank R. J. Archbold and C. J. K. Batty for pointing out the interest of the problems discussed here, and for correspondence and discussion which has been most helpful.

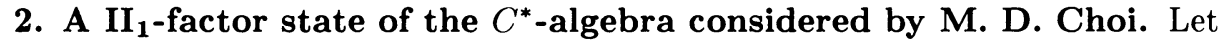
B be the $C^{*}$-algebra generated by two unitary operators $U, V$ on an infinitedimensional Hilbert space such that $U^{2}=V^{3}=I, E+U E U=1$, and $E+$ $V E V^{2}+V^{2} E V=1$ for some orthogonal projection $E$. No projections $F$ in the $C^{*}$-algebra generated by $U, V$ satisfies the above equations. $\mathbf{B}$ is called the Choi algebra. According to the remark after Theorem 2.8 in [2], $\mathbf{B}$ has a unique tracial state which is called the trace in this article. For the sake of giving a complete exposition we provide proofs for the trace to be a nonhyperfinite $\mathrm{II}_{1}$-factor state. It is shown in Corollary 2.7 in $[\mathbf{2}]$ that $\mathbf{B}$ is ${ }^{*}$-isomorphic to the reduced $C^{*}$-algebra of a discrete free group $G$ on two generators $u, v$ with $u^{2}=v^{3}=e$, the identity element in $G$.

LEMMA 2.1. Every element in $G$, except e, has an infinite conjugacy class.

ProOF. Let $w$ be an arbitrary element in $G$ other than $e . w$ can be viewed as a word composed of $u$ 's and $v$ 's in reduced form. Suppose that it both begins and ends with $u$ (or $v$, or $v^{-1}$ respectively). Then $\left\{(u v)^{k} w\left(v^{-1} u\right)^{k} \mid k=1,2, \ldots\right\}$ (or $\left\{(v u)^{k} w\left(u v^{-1}\right)^{k} \mid k=1,2, \ldots\right\}$ resp.) is an infinite set contained in the conjugacy class of $w$. Suppose that $w$ begins with $u$ and ends with $v$ (or $v^{-1}$ respectively). Then $z=v^{-1} w v$ (or $z=v w v^{-1}$ resp.) both begins and ends with $v^{-1}$ (or $v$ resp.). Hence $z$ has an infinite conjugacy class, and so does $w$. The remaining case where $w$ begins with $v$ (or $v^{-1}$ ) and ends with $u$ can be handled in a similar manner. Q.E.D.

Due to the work of Murray and von Neumann (Proposition 5 of III.7.6 in [4]) we know that the reduced von Neumann algebra $\mathbf{R}$ of $G$ is a $\mathrm{II}_{1}$-factor which is the weak-operator closure of $\mathbf{B}$. The unique normalized trace on $\mathbf{R}$, hence on $\mathbf{B}$, is a vector state induced by a cyclic vector $\chi_{e}$, the characteristic function of the 
identity element $e$ in $G$. Next, we show that $\mathbf{R}$ mentioned above is nonhyperfinite. It is sufficient to show $\mathbf{R}$ does not have property $\Gamma$ (for definition see Definition 2 of III.7.7 in [4]). By Lemma 12 of III.7.7 in [4], we need only show

LEMMA 2.2. The group $G$ in Lemma 2.1 contains a subset $X$ such that $X \cup$ $u X u=G \backslash\{e\}$ and $X, v X v^{-1}, v^{-1} X v$ are pairwise disjoint.

ProOF. Let $X$ consist of all reduced words in $G$ beginning with $u$. If $w \in G \backslash X$ and $w \neq e$, then $z=u w u$ is in $X$ and hence $w=u z u \in u X u$. $v X v^{-1}$ (or $v^{-1} X v$ respectively) consists of reduced words in $G$ beginning with $v$ (or $v^{-1}$ respectively) and hence $X, v X v^{-1}, v^{-1} X v$ are pairwise disjoint. Q.E.D.

Therefore we have shown

PROPOSITION 2.3. The unique normalized trace of $\mathbf{B}$ is a nonhyperfinite $\mathrm{II}_{1}$ factor state.

3. Irreducible representations of the Cuntz algebras $O_{2}$ on $l^{2}(G)$. Let $G$ be the free group on two generators $u, v$ with $u^{2}=v^{3}=e$ as in $\S 2$. In this section we construct subsets $F$ of $G$ satisfying the following two sets of conditions:

$$
F \cap(u F)=F \cap(v F)=\varnothing, \quad F \cup(u F)=G, \quad(v F) \cup\left(v^{2} F\right)=u F
$$

(3.2) there exists a sequence of words in $u, v,\left\{z_{i}\right\}$, such that $\bigcap\left(z_{i} F\right)=\{e\}$.

Firstly we show how an irreducible representation of $\mathrm{O}_{2}$ on $l^{2}(G)$ can be induced from such a subset $F$.

THEOREM 3.1. Let $F$ be a subset of $G$ satisfying both conditions (3.1) and (3.2). Then there is an irreducible representation of $\mathrm{O}_{2}$ on $l^{2}(G)$.

ProOF. Let $L$ be the left regular representation of $G$ on $l^{2}(G)$. Let $L u, L v$ be denoted by $U, V$ respectively, and note that $U^{2}=V^{3}=I$. Let $E$ be the projection of $l^{2}(G)$ onto the subspace generated by words in $F$. By condition (3.1), $E+U^{*} E U=I, E+V^{*} E V+V E V^{*}=I$. By Theorem 2.6 in [2], the $C^{*}$-algebra generated by $U, V$ and $E, C^{*}(U, V, E)$, is isomorphic with $O_{2}$. In the rest of this section we identify $O_{2}$ with $C^{*}(E, U, V)$. For any two reduced words $w_{1}, w_{2}$ in $u, v$, we let $\chi_{w_{i}}$ denote the characteristic function of $\left\{w_{i}\right\}, i=1,2$, and, for any operator $T$ in $\mathbf{B}\left(l^{2}(G)\right)$, the $C^{*}$-algebra of all bounded operators on $l^{2}(G)$, let $\left\langle T \chi_{w_{1}}, \chi_{w_{2}}\right\rangle$ be denoted by $T_{w_{1}, w_{2}}$. If $T \in O_{2}^{\prime}$, the commutant of $O_{2}$ in $\mathbf{B}\left(l^{2}(G)\right)$, then $T_{w_{1}, w_{2}}=T_{w w_{1}, w w_{2}}$ for all words $w$ in $u, v$. In fact,

$$
\begin{aligned}
T_{w w_{1}, w w_{2}} & =\left\langle T \chi_{w w_{1}}, \chi_{w w_{2}}\right\rangle=\left\langle T L \chi_{w}\left(\chi_{w_{1}}\right), L \chi_{w}\left(\chi_{w_{2}}\right)\right\rangle \\
& =\left\langle\left(L \chi_{w}\right)^{*} T\left(L \chi_{w}\right)\left(\chi_{w_{1}}\right), \chi_{w_{2}}\right\rangle=\left\langle T \chi_{w_{1}} \chi_{w_{2}}\right\rangle=T_{w_{1}, w_{2}}
\end{aligned}
$$

for any word $w$ in $u, v$, and $T \in O_{2}^{\prime}$. In particular, $T_{w, w}=T_{e, e}$ for all words $w$ in $u, v$. Finally, for two reduced words $w_{1} \neq w_{2}, T_{w_{1}, w_{2}}=T_{e, w_{1}^{-1} w_{2}}$ and let $w_{1}^{-1} w_{2}=w \neq e$. Then there is a positive integer $n$ such that $w \notin z_{n} F$ whereas $e \in z_{n} F$, i.e., $z_{n}^{-1} w \notin F$ and $z_{n}^{-1} \in F$. Hence $T_{e, w}=T_{z_{n}^{-1}}, z_{n}^{-1} w=\left\langle T \chi_{z_{n}^{-1}}, \chi_{z_{n}^{-1} w}\right\rangle=0$, for $\chi_{z_{n}^{-1}} \in E\left(l^{2}(G)\right) \Rightarrow T \chi_{z_{n}^{-1}} \in E\left(l^{2}(G)\right)$, and $\chi_{z_{n}^{-1} w}$ is in the orthogonal complement of $E\left(l^{2}(G)\right)$. This proves that $O_{2}$ acts irreducibly on $l^{2}(G)$. Q.E.D.

As is stated in the proof of Theorem 3.1, the left regular representation together with a choice of a subset $F$ gives rise to an irreducible representation $\pi$ of $\mathrm{O}_{2}$, which 
is also equivalent to the GNS representation induced by the vector state $\omega_{\chi_{e}}$, the trace on $\mathbf{B}$, and $\omega_{\chi_{e}} \circ \pi$ is regarded as an extension of the trace on $\mathbf{B}$ to $\mathrm{O}_{2}$. The corollary below follows.

\section{COROLlaRY 3.2. The trace on $\mathbf{B}$ has a pure state extension to $\mathrm{O}_{2}$.}

Throughout the rest of this section the complement of a subset $S$ of $G$ will be denoted by $S^{c}$, and both $\delta$ and $\sigma$ will be either 1 or 2 . In the proposition below we give a slightly different characterization of a subset $F$ of $G$ satisfying condition (3.1).

Proposition 3.3. Any subset $F$ of $G$ satisfying condition (3.1) also satisfies the following:

(1) $v^{\delta} F \subseteq F^{c}$ for $\delta=1,2$ and $u F=F^{c}$.

(2) $\left(u v^{\delta}\right) F \subseteq F$ for $\delta=1,2$.

(3) $\left(v^{\delta} u\right) F^{c} \subseteq F^{c}$ for $\delta=1,2$.

(4) If $w \in F$, then exactly one of $(v u) w$ and $\left(v^{2} u\right) w$ is in $F$.

$(4)^{\prime}$ If $w \in F^{c}$, then exactly one of $v w$ and $v^{2} w$ is in $F$.

In particular, conditions (4) and (4)' are equivalent under the assumption $u F=$ $F^{c}$. Furthermore the set of conditions (1) and (4), denoted by (3.3), is equivalent to $(3.1)$.

PROOF. (1) obviously follows from (3.1).

Since $v^{\delta} F \subseteq F^{c}=u F$, it follows that $\left(u v^{\delta}\right) F \subseteq u F^{c}=F$. Similarly $\left(v^{\delta} u\right) F^{c}=$ $\left(v^{\delta} u\right) u F=v^{\delta} F \subseteq F^{c}$. Then (2) and (3) are shown.

Let $w \in F^{c}=(v F) \cup\left(v^{2} F\right)$. If $w \in v F$, then $v^{2} w \in v^{2}(v F)=F$ and $v w \in$ $v^{2} F \subseteq F^{c}$. If $w \in v^{2} F$, then $v w \in F$ and $v^{2} w \in v F \subseteq F^{c}$. Thus (4)' is true.

Now we assume $u F=F^{c}$. Replacing $w$ by $u w$ in (4) one gets (4) and symmetrically replacing $w$ by $u w$ in (4) one gets (4)'.

Next we assume that a subset $F$ of $G$ satisfies (3.3). It is obvious that $F \cap$ $(u F)=\varnothing=F \cap(v F), F \cup(u F)=G$, and $\left(v^{\delta} F\right) \subseteq(u F)$. We need only show $(u F) \subseteq(v F) \cup\left(v^{2} F\right)$, which follows from $u F=F^{c}$ and condition (4). Q.E.D.

Here we set up some notation for the rest of this section. Let $A$ be the set of all words of the form

$$
\left(u v^{\delta_{n}}\right)\left(u v^{\delta_{n-1}}\right) \cdots\left(u v^{\delta_{1}}\right) \text { or } e
$$

where $\delta_{i}$ is either 1 or 2 for $1 \leq i \leq n$. It follows from (2) in Proposition 3.3 that if $F$ satisfies (3.1), and $w \in F$, then $x w$ must be in $F$ for all $x$ in $A$. Next, we let $\theta=\left\{n_{i}\right\}$ be a strictly increasing sequence of positive integers. For any (fixed) $w \in G$, we denote by $S(\theta, w)$ the set of all words of the form

$$
\left(v^{\delta} u\right)^{n_{p}} \ldots\left(v^{2} u\right)^{n_{2}}(v u)^{n_{1}} w, \quad p=0,1,2, \ldots,
$$

where $\delta$ is 2 for even $p$ and $\delta$ is 1 for odd $p$. The length of a reduced word in $u, v$ is the number of characters in that word.

Proposition 3.4. Suppose that a subset $F$ of $G$ satisfies (3.1) and $S(\theta, v) \subseteq$ $F$. Then $v$ is the only word $w$ in $u, v$ such that $S(\theta, w) \subseteq F$.

In order to prove Proposition 3.4 we need the following lemmas in which $F$ is assumed to satisfy $(3.1)$ and $S(\theta, v) \subseteq F$. 
LEMMA 3.5. Let $p=0,1,2, \ldots, \delta$ be 2 for even $p$ and 1 for odd $p$, and $\bar{\delta}=3-\delta$. Then the words of the form

$$
\left(v^{\bar{\delta}} u\right)^{k}\left(v^{\delta} u\right)^{n_{p}} \cdots(v u)^{n_{1}} v, \quad 0 \leq k<n_{p+1}
$$

are in $F$.

ProOF. Observe that $w=\left(v^{\bar{\delta}} u\right)^{n_{p+1}}\left(v^{\delta} u\right)^{n_{p}} \ldots(v u)^{n_{1}} v$ is in $F$. Then straightforward applications of condition (2) in Proposition 3.3 to $w$ will yield the desired result. Q.E.D.

LEMMA 3.6. Let $\delta$ be 2 for even $p$ and 1 for odd $p, p=1,2, \ldots$ Consider any word $w=\left(v^{\delta} u\right)^{m_{p}} \cdots\left(v^{2} u\right)^{m_{2}}(v u)^{m_{1}} v$, where $m_{j} \geq 0,1 \leq j \leq p$, and $m_{i} \neq n_{i}$ for at least one $i$ with $1 \leq i \leq p-1$, or $m_{i}=n_{i}$ for all $1 \leq i \leq p-1$ and $m_{p}>n_{p}$. Words thus obtained are not in $F$.

PROOF. Let $k$ be the smallest integer such that $m_{k} \neq n_{k}$ in the word described above, and denote it by $w_{0}$. Suppose $w_{0} \in F$. We will deduce a contradiction from this supposition. In what follows we let $\sigma, \rho$ be 1 or 2 and $\bar{\rho}=3-\rho, \bar{\sigma}=3-\sigma$.

Case 1. Suppose $m_{k}<n_{k}$. This implies that $k<p$. By Lemma 3.5 we know that both $w_{1}\left(v^{\sigma} u\right)^{m_{k}}\left(v^{\bar{\sigma}} u\right)^{n_{k-1}} \cdots(v u)^{n_{1 v}}$ and $\left(v^{\sigma} u\right) w_{1}$ are in $F$. Several applications of (2) in Proposition 3.3 to $w_{0}$ show that $\left(v^{\bar{\sigma}} u\right) w_{1}$ is also in $F$. This is a contradiction to (4) in Proposition 3.3.

Case 2. Suppose $m_{k}>n_{k}$. Applying (2) in Proposition 3.3 to $w_{0}$ a number of times, one sees that both $s=\left(v^{\rho} u\right)^{n_{k}} \cdots(v u)^{n_{1}} v$ and $\left(v^{\rho} u\right) s$ are in $F$. By Lemma $3.5\left(v^{\bar{\rho}} u\right) s$ is also in $F$. Again this is a contradiction to (4) in Proposition 3.3. Q.E.D.

LEMMA 3.7. Let $\left\{m_{1}, m_{2}, \ldots, m_{k}\right\}$ be a nonempty set of positive integers. Then words of the form

$$
\left(v^{\sigma} u\right)^{n_{p}} \cdots\left(v^{2} u\right)^{n_{2}}(v u)^{n_{1}}\left(v^{2} u\right)^{m_{k}} \cdots\left(v^{\delta} u\right)^{m_{1}} v \quad \text { with } p \geq 2,
$$

where $\sigma$ is 2 for even $p$ and 1 for odd $p$, and $\delta$ is 1 for even $k$ and 2 for odd $k$, are not in $F$.

PROOF. Since $\left\{n_{i}\right\}$ is an increasing sequence and $p \geq 2$, the adjoint sequence $\left\{m_{1}, m_{2}, \ldots, m_{k}, n_{1}, n_{2}, \ldots, n_{p}\right\}$ must differ from $\left\{n_{1}, \ldots, n_{k}, \ldots, n_{p+k}\right\}$ at the $(k+1)$ st term. If $k$ is odd, the words described in Lemma 3.7 are of the form $\left(v^{\sigma} u\right)^{n_{p}} \cdots(v u)^{n_{1}}\left(v^{2} u\right)^{m_{k}} \cdots\left(v^{2} u\right)^{m_{1}} v$ and thus is not in $F$ by Lemma 3.6. If $k$ is even, the words described in Lemma 3.7 are of the form

$$
\left(v^{\sigma} u\right)^{n_{p}} \ldots(v u)^{n_{1}}\left(v^{2} u\right)^{m_{k}} \cdots(v u)^{m_{1}} v
$$

in which $n_{1} \neq n_{k+1}$. Hence they are not in $F$ by Lemma 3.6. Q.E.D.

REMARK 3.8. Actually Lemmas 3.5, 3.6, and 3.7 remain true for those subsets $F$ of $G$ satisfying (2) and (4) in Proposition 3.3 and $S(\theta, v) \subseteq F$.

LEMMA 3.9. No reduced words beginning with $v$ or $v^{2}$ and ending with $u$ or $v^{2}$ can be in $F$.

ProOF. From Lemma 3.5 we see $v \in F$ and hence $v^{2} v=e \in F^{c}, v^{2}=v \cdot v \in F^{c}$ and $u=u \cdot e \in F$, by (1) in Proposition 3.3. A reduced word, beginning with $v^{\delta}$ and ending with $u$, is of even length, say $2 n, n \geq 0$. If such a word is in $F$, then 
applying (2) in Proposition $3.3 n$ times, one gets $e \in F$, a contradiction. Hence such a word is not in $F$. A reduced word, beginning with $v^{\delta}$ and ending with $v^{2}$, is of odd length, say $1+2 n, n \geq 0$. If such a word is in $F$, then applying (2) in Proposition $3.3 n$ times, one gets $v^{2} \in F$, a contradiction. Hence such a word is not in $F$. Q.E.D.

PROOF OF PROPOSITION 3.4.

Case 1. Let $w$ be a reduced word beginning with $v^{\sigma}, \sigma=1$ or 2 , but different from $v$.

Consider $w_{1}=\left(v^{2} u\right)^{n_{2}}(v u)^{n_{1}} w$ which is not in $F$ if $w$ ends with $v^{2}$ or $u$ by Lemma 3.9. While $w$ ends with $v, w_{1}$ cannot be in $F$ by Lemma 3.7. Thus $S(\theta, w) \nsubseteq F$.

Case 2. Let $w$ be a reduced word beginning with $u$ of length $p$.

Consider the word $w_{2}=\left(v^{2} u\right)^{n_{2 p}} \ldots(v u)^{n_{1}} w$. In its reduced form $w_{2}$ begins with $v^{2}$ and ends with either $u$ or $v^{2}$, hence not in $F$, or $v$. When it ends with $v$, it will be like words in Lemma 3.6, hence not in $F$. Thus $S(\theta, w) \nsubseteq F$. Q.E.D.

PROPOSITION 3.10. Suppose that a subset $F$ of $G$ satisfies (3.1) and $S(\theta, v) \subseteq$ $F$. Then $F$ satisfies (3.2) as well.

PROOF. Let $z_{i}=v^{2}\left(u v^{2}\right)^{n_{1}}(u v)^{n_{2}} \cdots\left(u v^{\sigma}\right)^{n_{i}}$ for $i=1,2, \ldots$, where $\sigma=2$ if $i$ is odd and $\sigma=1$ if $i$ is even. We need only show $\bigcap_{i}\left(z_{i} F\right)=\{e\}$. Since $z_{i}^{-1}=\left(v^{\bar{\sigma}} u\right)^{n_{i}} \cdots(v u)^{n_{1}} v$, where $\bar{\sigma}=3-\sigma$, is in $F$ for all $i=1,2, \ldots$, it follows that $\{e\} \subseteq \bigcap_{i}\left(z_{i} F\right)$. The presence of a word $w$ in $\bigcap_{i}\left(z_{i} F\right)$ implies that $z_{i}^{-1} w$ is in $F$ for all $i=1,2, \ldots$, which is the same as $S(\theta, v w) \subseteq F$. By Proposition 3.4 we have $v w=v$ and hence $w=e$. Thus $\{e\}=\bigcap_{i}\left(z_{i} F\right)$. Q.E.D.

Following from Proposition 3.10 and the remark preceding Proposition 3.4, it is only natural to try to construct a subset $F$ satisfying (3.1) and (3.2) by the procedure below:

Given an increasing sequence $\theta=\left\{n_{i}\right\}$ of positive integers, let $F_{\theta}$ be the subset of $G$ consisting of words in the form of $x y$, with $x \in A$ and $y \in S(\theta, v)$. (For the definition of $A$ and $S(\theta, v)$, see the remark before Proposition 3.4.) It is obvious that $S(\theta, v) \subseteq F_{\theta}$, because $e \in A$. Thus $v \in F_{\theta}$ and $u=\left(u v^{2}\right) v \in F_{\theta}$. Many examples of such subsets $F_{\theta}$ can be obtained. In a forthcoming paper we will show that all these uncountable irreducible representations of $\mathrm{O}_{2}$ constructed from $\theta$ 's with different tails are inequivalent. By Proposition 3.10 only the validity of (3.1) for $F_{\theta}$ remains to be shown.

Proposition 3.11. $F_{\theta}$ satisfies (3.1).

PrOOF. By Proposition 3.3 it is sufficient to show that $F_{\theta}$ satisfies conditions (1) and (4) in Proposition 3.3. Actually $F_{\theta}$ is the union of the following disjoint subsets:

$$
\begin{aligned}
& F_{1}=\{\text { elements in Lemma } 3.5\} \\
& \begin{array}{c}
F_{2}=\left\{\left(u v^{\delta_{n}}\right) \cdots\left(u v^{\delta_{1}}\right)\left(u v^{\delta} u\right)\left(v^{\bar{\delta}} u\right)^{k}\left(v^{\delta} u\right)^{n_{p}} \cdots\left(v^{2} u\right)^{n_{2}}(v u)^{n_{1}} v\right. \\
\text { with } 0 \leq k<n_{p+1} \text { for some } n, p \geq 0, \text { where } \delta_{i}=1 \text { or } 2 \text { for } i= \\
1, \ldots, n, \text { and } \delta=2 \text { if } p \text { is even and } \delta=1 \text { if } p \text { is odd, } \bar{\delta}=3-\delta\} \\
F_{3}=\left\{\left(u v^{\delta_{n}}\right) \cdots\left(u v^{\delta_{1}}\right)\left(u v^{2}\right) \text { or }\left(u v^{\delta_{n}}\right) \cdots\left(u v^{\delta_{1}}\right) u,\right. \\
\left.n=0,1,2, \ldots, \text { where } \delta_{i}=1 \text { or } 2 \text { for } i=1, \ldots, n\right\} .
\end{array}
\end{aligned}
$$


First of all, we check that conditions (2) and (4) in Proposition 3.3 hold for $F_{\theta}$, and then Lemmas $3.5,3.6$, and 3.7 can apply to $F_{\theta}$. Finally we will show that condition (1) in Proposition 3.3 holds for $F_{\theta}$.

Let $w$ be in $F_{1}$ and $w=\left(v^{\bar{\delta}} u\right)^{k}\left(v^{\delta} u\right)^{n_{p}} \cdots(v u)^{n_{1}} v, 0 \leq k<n_{p+1}, \bar{\delta}=3-\delta$. Thus $\left(v^{\bar{\delta}} u\right) w \in F_{\theta}$ and $\left(v^{\delta} u\right) w \notin F_{j}$ for $j=1,2,3$, hence $w$ is not in $F_{\theta}$. For $k>0$, $\left(u v^{\delta}\right) w \in F_{1}$ and $\left(u v^{\bar{\delta}}\right) w \in F_{2}$. For $k=0,\left(u v^{\bar{\delta}}\right) w \in F_{1}$ and $\left(u v^{\delta}\right) w \in F_{2}$.

Let $w$ be in $F_{2}$. Thus $\left(v^{\bar{\delta}_{n}} u\right) w \in F_{2},\left(v^{\delta_{n}} u\right) w \notin F_{\theta}$ if $n \neq 0$, and $\left(v^{\delta} u\right) w \in F_{1}$, $\left(v^{\bar{\delta}} u\right) w \notin F_{\theta}$ if $n=0$, by the description of $F_{1}, F_{2}, F_{\theta}$. It is easy to see that $\left(u v^{\delta}\right) w \in F_{2}$ for $\delta=1,2$.

Now let $w$ be in $F_{3}$. It is obvious that $\left(u v^{\delta}\right) w \in F_{3}$ for $\delta=1,2 .\left(v^{\bar{\delta}_{n}} u\right) w \in F_{3}$ and $\left(v^{\delta_{n}} u\right) w \notin F_{\theta}$ if $n>0$. Otherwise $\left(v^{2} u\right)\left(u v^{2}\right) \in F_{\theta},(v u)\left(u v^{2}\right) \notin F_{\theta}$ and $\left(v^{2} u\right) u \notin F_{\theta},(v u) u \in F_{\theta}$. Hence condition (2) and (4) in Proposition 3.3 hold for $F_{\theta}$.

Next we check that condition (1) in Proposition 3.3 holds for $F_{\theta}$. Firstly we show $v^{\delta} F_{\theta} \subseteq F_{\theta^{c}}$ for $\delta=1,2$.

Let $w \in F_{3}$. Then $v^{\delta} w \in F_{\theta}^{c}$, for $v^{\delta} w$ in its reduced form begins with $v^{\delta}$ and ends with $v^{2}$ or $u$, hence is not in $F_{\theta}$ by the description of $F_{1}, F_{2}, F_{3}$.

Let $w \in F_{2}$. Then $v^{\delta} w$ is like a word described in Lemma 3.6 and hence is not in $F_{\theta}$.

Let $w \in F_{1}$. One checks by inspection that for $\delta=1$ or $2, v^{\delta} w \notin F_{j}, j=1,2,3$, and hence $v^{\delta} w \notin F_{\theta}$ for $\delta=1,2$.

Secondly we check $u F_{\theta} \subseteq F_{\theta^{c}}$.

Let $w \in F_{1}$. Then by inspection $u w$ does not belong to $F_{j}$ for $j=1,2,3$ and hence $u w \in F_{\theta^{c}}$.

For any word $w$ in $F_{3}$, except $w=u$, uw begins with $v^{\delta}, \delta=1$ or 2 , and ends with $v^{2}$ or $u$, hence not in $F_{\theta}$. In case $w=u, u w=e$ is not in $F_{\theta}$.

For any word $w$ in $F_{2}$, except $w=v, u w$ is like a word described in Lemma 3.6, hence not in $F_{\theta}$. In case $w=v, u v$ is not in $F_{\theta}$.

Finally, we check $u F_{\theta}^{c} \subseteq F_{\theta}$.

Case 1. $w$ begins with $v^{\delta}, \delta=1$ or 2 .

If $w$ ends with $u$ or $v^{2}$, then $u w \in F_{3}$. Otherwise, $w$ must be of the form $\left(v^{\delta} u\right)^{r_{s}} \ldots\left(v^{2} u\right)^{r_{2}}(v u)^{r_{1}} v$ for some $s>0$ and $r_{i} \geq 0$ for $i=1, \ldots, s$. We note that one of $r_{i}$ 's must be nonzero, for otherwise $w=v \in F_{\theta}$. Actually we may assume that $r_{i}>0$ for $2 \leq i \leq s$. If $r_{1}=0$, then $s \geq 2$ and $u w$ is in $F_{2}$, as an element of the general form in $F_{2}$ with $p=k=0$. Suppose $r_{1} \neq 0$. Since $w \notin F_{\theta}$, there is at least one $i$ such that $r_{i} \neq n_{i}$. Let $j$ be the smallest integer such that $r_{j} \neq n_{j}$. If $r_{j}>n_{j}$, then $u w$ is in $F_{2}$, as an element of the general form in $F_{2}$ with $k=0$, $p>0$. If $r_{j}<n_{j}$, one sees that $u w$ is in $F_{2}$ as an element of the general form with $k=r_{j}$, because $w \notin F_{\theta}, j<s$.

Case 2. $w$ begins with $u$.

Then $w$ must end with $v$ and is of the form $u\left(v^{\delta} u\right)^{r_{s}} \ldots\left(v^{2} u\right)^{r_{2}}(v u)^{r_{1}} v$ for some $s \geq 0$ and $r_{i} \geq 0$ for $i=1, \ldots, s$. We may assume $s>0$, and one of the $r_{i}$ 's is nonzero, for otherwise $w=u v$ and $u w \in F_{\theta}$. Let $w_{1}$ be $u w$. Then $w_{1}$, in its reduced form beginning with $v^{\delta}$ and ending with $v$, cannot be in $F_{\theta}^{c}$, for otherwise $u w_{1}=w$ would be in $F_{\theta}$ by Case 1 above. Hence $w_{1}=u w$ must be in $F_{\theta}$. Q.E.D. 


\section{REFERENCES}

1. R. J. Archbold and C. J. K. Batty, Extensions of factorial states of $C^{*}$-algebras, J. Funct. Anal. 63 (1985), 86-100.

2. M. D. Choi, $A$ simple $C^{*}$-algebra generated by two finite order unitaries, Canad. J. Math. 31 (1979), 867-880.

3. J. Cuntz, Simple $C^{*}$-algebra generated by isometries, Comment. Math. Phys. 57 (1977), 173185.

4. J. Dixmier, Les algèbres d'opérateurs dans l'espace Hilbertien, Gauthier-Villars, Paris, 1969.

5. D. E. Evans, $O_{n} O_{n}$, Publ. Res. Inst. Math. Sci. 16 (1980), 915-927.

6. R. Longo, Solution of the factorial Stone-Weierstrass conjecture. An application of standard split $W^{*}$-inclusions, Invent. Math. 76 (1984), 145-155.

7. S. Popa, Constructing semiregular maximal abelian *-subalgebras in factors, Invent. Math. 65 (1984), 157-161.

8. S. Sakai, Talk given in Wabash International Conference on Banach Spaces in 1973, unpublished.

9. S.-k. Tsui, Central sequences associated with a state, Proc. Amer. Math. Soc. 82 (1981), 76-81.

10. S. L. Woronowicz, On the purification of factor states, Comm. Math. Phys. 78 (1972), 221-235.

Department of Mathematical Sciences, Tel-Aviv University, Tel-Aviv, ISRAEL (Current address of A. Lazar)

Department of Mathematical Sciences, Oakland University, Rochester, MICHIGAN 48063 (Current address of S.-k. Tsui and S. Wright) 